\title{
A NOTE ON PERMANENTS
}

\author{
BY \\ MORTON ABRAMSON
}

Let $A=\left(a_{i j}\right)$ be an $m \times n$ matrix and let $K=\left\{s_{1}, \ldots, s_{k}\right\}$ be a $k$-subset from $\{1,2, \ldots, n\}$. For $0 \leq t \leq k \leq n$ define the $(t, K)$-permanent of $A$ to be

$$
\operatorname{per}_{(t, K)}(A)=\sum a_{1 i_{1}} a_{2 i_{2}} \ldots a_{m i_{m}}
$$

the summation taken over all $m$-tuples $\left(i_{1}, i_{2}, \ldots, i_{m}\right)$ (repetitions allowed) of $1,2, \ldots, n$ each containing exactly $t$ distinct entries from $K$ and any number of distinct entries from the remaining $n-k$ integers. For example, $(4,4,7,1,1,2)$, $(4,4,6,6,6,5)$ are 6-tuples, each containing exactly two distinct entries from $K=\{2,4,5\}$ for $n \geq 7$. We define the $t$-permanent of $A$ to be the case $K=\{1,2, \ldots, n\}$ and write

$$
\operatorname{per}_{t}(A)=\operatorname{per}_{(t, K)}(A), \quad K=\{1,2, \ldots, n\},
$$

each $m$-tuple $\left(i_{1}, \ldots, i_{m}\right)$ in the summation in (1) containing exactly $t$ distinct entries of $\{1,2, \ldots, n\}$. When $t=m=n,(2)$ is the "ordinary" permanent of a square matrix (see [1] for a survey article), while $t=m \leq n$ is the generalization to rectangular matrices described by Ryser [3].

Let $A_{r}$ denote a matrix obtained from $A$ by replacing $r$ of the $k$ columns $s_{1}, s_{2}, \ldots, s_{k}$ of $A$ by zeros, $S\left(A_{r}\right)$ the product of the row sums of $A_{r}$ and $\sum S\left(A_{r}\right)$ the sum of all the $\left(\begin{array}{l}k \\ r\end{array}\right)$ numbers $S\left(A_{r}\right)$. Then $\operatorname{per}_{(t, K)}(A)$ can be evaluated by

$$
\operatorname{per}_{(t, k)}(A)=\sum_{i=0}^{t}(-1)^{k}\left(\begin{array}{c}
k-t+i \\
i
\end{array}\right) \sum S\left(A_{k-t+i}\right)
$$

and hence

$$
\operatorname{per}_{t}(A)=\sum_{i=0}^{t}(-1)^{i}\left(\begin{array}{c}
n-t+i \\
i
\end{array}\right) \sum S\left(A_{n-t+i}\right)
$$

Formula (4) in the case $m=t \leq n$ was first observed by Ryser [3, p. 26] and his elegant proof (based on the Principle of Inclusion and Exclusion) suffices, with minor modification, to establish (3), so we omit the proof.

When $t=k$, (3) becomes

$$
\operatorname{per}_{(k, K)}(A)=\sum_{i=0}^{k}(-1)^{i} \sum S\left(A_{i}\right)
$$

Let ${ }_{t} A$ be a submatrix of $A$ obtained by deleting $n-t$ columns of $A$. It follows that (6)

$$
\operatorname{per}_{t}(A)=\sum \operatorname{per}_{t}\left({ }_{t} A\right)
$$

Received by the editors March 18, 1970. 
the summation taken over all the $\left(\begin{array}{c}n \\ t\end{array}\right)$ choices of ${ }_{t} A$. In the case $t=m \leq n, \operatorname{per}_{m}(A)$ is therefore equal to the sum of permanents of square submatrices of order $m$. It is clear that

$$
\sum_{t=1}^{n} \operatorname{per}_{t}(A)=A_{0}
$$

$A_{0}$ being the product of the row sums of $A$. In the case $m<t \leq n, \operatorname{per}_{t}(A)=0$.

We describe several of many possible applications.

Suppose that $m$ distinct objects $d_{1}, \ldots, d_{m}$ are to be distributed into $n$ distinct cells $c_{1}, \ldots, c_{n}$. An $m \times n(0,1)$ matrix $A=\left(a_{i j}\right)$ can be interpreted as describing restrictions on the distribution, namely object $d_{i}$ can be placed in cell $c_{j}$ if and only if $a_{i j}=1$. Suppose furthermore that $t$ is given, and we insist that exactly $t$ cells are nonempty. Then the number of such distributions (each satisfying conditions described by $A$ and exactly $t$ cells nonempty) is $\operatorname{per}_{t}(A)$. For a fixed subset $K=\left\{s_{1}, \ldots, s_{k}\right\}$ of $\{1,2, \ldots, n\}, \operatorname{per}_{(t, k)}(A)$ is the number of distributions each satisfying conditions described by $A$ and exactly $t$ of the cells $c_{s_{1}}, c_{s_{2}}, \ldots, c_{s_{k}}$ nonempty.

It is easy to see that $\operatorname{per}_{n}(A)>0$ if and only if $n$ is equal to the term rank of $A$, i.e., there is a way of distributing the objects with no cell empty if and only if the maximal number of 1's in $A$, no two in a row or column, is $n$. Equivalently, $\operatorname{per}_{n}(A)>0$ if and only if the $n$ subsets of $\{1,2, \ldots, n\}$ whose incidence matrix is the transpose of $A$ have a system of distinct representatives.

If any object is permitted into any cell, the matrix $A$ becomes $J_{m, n}$ all of whose entries are 1. Then the number of distributions each with exactly $t$ of the first $k$ cells nonempty (the remaining $n-k$ cells may or may not be empty) is, by (3) with $K=\{1,2, \ldots, k\}, k \leq n$,

$$
\begin{aligned}
\operatorname{per}_{(t, K)}\left(J_{m, n}\right) & =\sum_{i=0}^{t}(-1)^{i}\left(\begin{array}{c}
k-t+i \\
i
\end{array}\right)\left(\begin{array}{c}
k \\
k-t+i
\end{array}\right)(t+n-i-k)^{m} \\
& =\left(\begin{array}{c}
k \\
t
\end{array}\right) \sum_{i=0}^{t}(-1)^{i}\left(\begin{array}{c}
t \\
i
\end{array}\right)(t+n-i-k)^{m} .
\end{aligned}
$$

In the case $t=k,(8)$ becomes,

$$
\operatorname{per}_{(k, K)}\left(J_{m, n}\right)=\sum_{i=0}^{t}(-1)^{i}\left(\begin{array}{l}
k \\
i
\end{array}\right)(n-i)^{m}= \begin{cases}0 & \text { if } 1 \leq m<k \\
k ! & \text { if } m=k\end{cases}
$$

Formula (9) may also be obtained directly using the principle of inclusion and exclusion or by noting that $\operatorname{per}_{(k, K)}\left(J_{m, n}\right)$ is the coefficient of $x^{m} / m$ ! in the expression $\left(e^{x}-1\right)^{k}\left(e^{x}\right)^{n-k}, k \leq n$. The number of distributions with none of the $n$ cells empty is formula (9) with $k=n$, namely the well-known formula

$$
\operatorname{per}_{n}\left(J_{m, n}\right)=\sum_{i=0}^{n-1}(-1)^{i}\left(\begin{array}{l}
n \\
i
\end{array}\right)(n-i)^{m}= \begin{cases}0 & \text { if } 1 \leq m<n \\
n ! & \text { if } m=n\end{cases}
$$

This is also the number of ordered partitions of a finite set of $m$ objects into $n$ disjoint nonempty subsets, while the number of unordered partitions is

$$
S(m, n)=(1 / n !) \operatorname{per}_{n}\left(J_{m, n}\right)
$$


the numbers $S(m, n)$ usually being called Stirling numbers of the second kind [2]. By (6) and (7) we have

$$
\sum_{t=1}^{n} \operatorname{per}_{t}\left(J_{m, n}\right)=\sum_{t=1}^{n}\left(\begin{array}{c}
n \\
t
\end{array}\right) \operatorname{per}_{t}\left(J_{m, t}\right)=n^{m}
$$

and therefore using (11)

$$
\sum_{t=1}^{n} S(m, t)(n)_{t}=n^{m}, \quad(n)_{t}=n(n-1) \ldots(n-t+1), \quad \text { (see [2]). }
$$

If object $d_{i}$ is placed into cell $c_{j}$ with probability $p_{i j}$, then, letting $P=\left(p_{i j}\right)$, the probability that exactly $t$ of the cells are nonempty is $\operatorname{per}_{t}(P)$.

A generalization of Montmort's "problème des rencontres" is obtained by taking $A=\left(a_{i j}\right)$ with $a_{i i}=0, i=1,2, \ldots, k, k \leq \min (m, n)$ and $a_{i j}=1$ otherwise. Then the number of distributions of $m$ objects into the $n$ cells such that for $i=1$, $2, \ldots, k$ cell $c_{i}$ is nonempty and object $d_{i}$ is not in cell $c_{i}$, is by (5) with $K=\{1,2$, $\ldots, k\}$,

$$
\operatorname{per}_{(k, K)}(A)=D_{k}(m, n)=\sum_{i=0}^{k}(-1)^{i}\left(\begin{array}{l}
k \\
i
\end{array}\right)(n-i)^{m-k+i}(n-i-1)^{k-i},
$$

with $D_{0}(m, n)=n^{m}$ while,

$$
\begin{aligned}
D(m, n) & =\operatorname{per}_{n}(A)=D_{n}(m, n) \\
& =\sum_{i=0}^{n}(-1)^{i}\left(\begin{array}{l}
n \\
i
\end{array}\right)(n-i)^{m-n+i}(n-i-1)^{n-i}, \quad m \geq n,
\end{aligned}
$$

is the number of distributions with none of the cells empty and object $d_{i}$ not in cell $c_{i}, i=1,2, \ldots, n$. Thus, the ordinary rencontres numbers are given by

$$
D(n, n)=\sum_{i=0}^{n}(-1)^{i}\left(\begin{array}{c}
n \\
i
\end{array}\right)(n-i)^{i}(n-i-1)^{n-i},
$$

an expression noted by Ryser [3, p. 28]. It is easily seen that

$$
\sum_{t=1}^{n}\left(\begin{array}{l}
n \\
t
\end{array}\right) D(m, t)=(n-1)^{n} n^{m-n}
$$

The expression for $D(m, n)$ given by (15) may be easily obtained directly using the principle of inclusion and exclusion. Denote by $g(m, n)$ the number of distributions of $m$ objects into $n$ cells with object $d_{i}$ not in cell $c_{i}, i=1, \ldots, n$. Clearly

$$
g(m, n)=(n-1)^{n} n^{m-n} \text { and } D(m, n)=\sum_{i=0}^{n}(-1)^{i}\left(\begin{array}{c}
n \\
i
\end{array}\right) g(m, n-i), \quad m \geq n,
$$

giving (15). Similarly (14) may be obtained directly. A second expression may be obtained for $D(m, n)$ (also by the use of inclusion and exclusion), namely

$$
\begin{aligned}
D(m, n) & =\sum_{k=0}^{n}(-1)^{k}\left(\begin{array}{l}
n \\
k
\end{array}\right) \operatorname{per}_{(n-k, K)}\left(J_{m-k, n}\right), \quad K=\{1,2, \ldots, n-k\}, \\
& =\sum_{k=0}^{n}(-1)^{k}\left(\begin{array}{l}
n \\
k
\end{array}\right) \sum_{j=0}^{n-k}(-1)^{j}\left(\begin{array}{c}
n-k \\
j
\end{array}\right)(n-j)^{m-k},
\end{aligned}
$$


so

$$
\begin{aligned}
D(n, n) & =\sum_{k=0}^{n}(-1)^{k}\left(\begin{array}{l}
n \\
k
\end{array}\right) \sum_{j=0}^{n-k}(-1)^{j}\left(\begin{array}{c}
n-k \\
j
\end{array}\right)(n-j)^{n-k} \\
& =\sum_{k=0}^{n}(-1)^{k}\left(\begin{array}{l}
n \\
k
\end{array}\right)(n-k) !=n ! \sum_{k=0}^{n}(-1)^{k} / k ! .
\end{aligned}
$$

The last expression of (19) is the most common one given for the rencontres numbers [3, p. 23]. Denote by $D(m, n, r), m \geq n$, the number of $m$-permutations of $1,2, \ldots, n$, repetitions allowed, such that each of the $n$ integers appears at least once in each permutation with exactly $r$ of them being in natural position. Then $D(m, n, 0)=D(m, n)$ and

$$
D(m, n, r)=\left(\begin{array}{l}
n \\
r
\end{array}\right) D_{n-r}(m-r, n), \quad r=0,1, \ldots, n,
$$

while

$$
\sum_{r=0}^{n} D(m, n, r)=n ! S(m, n)
$$

the numbers $D_{k}(m, n)$ and $S(m, n)$ given by (14) and (11) respectively.

In the case $A$ is a $(0,1)$ matrix with $m<n$ and all entries on the main diagonal zero and one elsewhere,

$$
\operatorname{per}_{n}(A)=\sum_{k=0}^{n-1}(-1)^{k} \sum_{u=0}^{k}\left(\begin{array}{l}
m \\
u
\end{array}\right)\left(\begin{array}{c}
n-m \\
k-u
\end{array}\right)(n-k)^{u}(n-k-1)^{m-u}=0 .
$$

With regard to matching problems we have the following. Let $A_{1}, \ldots, A_{m}$ be subsets of an $n$-set $S$ and define a system of $t$-representatives of $\left(A_{1}, \ldots, A_{m}\right)$ to be an $m$-tuple $\left(a_{1}, \ldots, a_{m}\right)$ containing exactly $t$ distinct elements of $S$ with $a_{i} \in A_{i}$, $i=1, \ldots, m$. Then the number of systems of $t$-representatives of $\left(A_{1}, \ldots, A_{m}\right)$ is equal to the $t$-permanent of the corresponding $(0,1)$ incidence matrix of size $m$ by $n$. Putting $t=\min (m, n)$, we have the number of systems of maximum distinct representatives and when $t=m \leq n$, the number of systems of distinct representatives $[3$, p. 54, Theorem 4.1].

\section{REFERENCES}

1. Marvin Marcus and Henryk Minc, Permanents, Amer. Math. Monthly, 72 (1965), 577-591.

2. J. Riordan, An introduction to combinatorial analysis, Wiley, New York, 1958.

3. Herbert John Ryser, Combinatorial mathematics, Carus Math. Monograph No. 14, 1963.

YORK UNIVERSITY, TORONIO, ONTARIO 